\title{
Compression stockings reduce the incidence of hypotension but not that of cerebral desaturation events in the beach-chair position: a randomized controlled trial
}

\author{
Jae Hee Woo ${ }^{1}$, Youn Jin Kim ${ }^{1}$, Ji-sun Jeong ${ }^{1}$, Ji Seon Chae ${ }^{1}$, Young Rong Lee ${ }^{2}$, \\ and Jin Young Chon ${ }^{2}$
}

Department of Anesthesiology and Pain Medicine, ${ }^{1}$ Ewha Womans University College of Medicine, ${ }^{2}$ Yeouido St. Mary's Hospital, College of Medicine, The Catholic University of Korea, Seoul, Korea

\begin{abstract}
Background: The beach-chair position (BCP) results in decreases in venous return, cardiac output, and cerebral perfusion pressure. In this randomized, prospective study, we investigated whether applying thigh-high compression stockings affected the maintenance of regional cerebral tissue oxygen saturation $\left(\mathrm{rSO}_{2}\right)$ in the BCP.

Methods: Patients undergoing orthopedic surgery in the BCP under general anesthesia were included and assigned randomly to the control or the compression stocking group. Appropriately sized thigh-high compression stockings were applied to the patients in the stockings group. All patients were tilted, up to $45^{\circ}$, throughout the operation. Non-invasive blood pressure, invasive arterial blood pressure zeroed at the external auditory meatus, and $\mathrm{rSO}_{2}$ were recorded.

Results: Data were analyzed from 19 patients per group. In the $\mathrm{BCP}$, the values of $\mathrm{rSO}_{2}$ and blood pressure decreased significantly compared with those at baseline, with no significant difference between the groups. The incidences of cerebral desaturation events (CDEs) were similar between the groups; however, that of hypotension was significantly lower in the compression stocking group. During $36 \mathrm{CDEs}$, the levels of $\mathrm{rSO}_{2}$ and blood pressure decreased significantly compared with those at baseline in both groups. No significant correlation was found between $\mathrm{rSO}_{2}$ and blood pressure.

Conclusions: Thigh-high compression stockings reduced the incidence of hypotension but not that of CDEs. Our results suggest that other factors, beyond hypotension itself, contribute to CDEs and in other words, efforts just to reduce the incidence of hypotension may not mainly contribute to a reduction of CDEs occurrence in the BCP under general anesthesia.
\end{abstract}

Keywords: Beach chair position; Cerebral perfusion pressure; Compression stockings; Hypotension; Near-infrared spectroscopy; Shoulder surgery.

Corresponding author: Youn Jin Kim, M.D., Ph.D.

Department of Anesthesiology and Pain Medicine, Ewha Womans University College of Medicine, 1071, Anyangcheon-ro, Yangcheon-gu, Seoul 07985, Korea

Tel: 82-2-2650-5285, Fax: 82-2-2655-2924, Email: ankyj@ewha.ac.kr

ORCID: https://orcid.org/0000-0001-9189-5839

It was presented at the Euroanaesthesia 2016, May 2016, ExCeL London, London, UK.

Received: May 11, 2017. Revised: June 19, 2017. Accepted: June 21, 2017.

Korean J Anesthesiol 2018 April 71(2): 127-134

https://doi.org/10.4097/kjae.2018.71.2.127

(c) This is an open-access article distributed under the terms of the Creative Commons Attribution Non-Commercial License (http://creativecommons.org/ licenses/by-nc/4.0/), which permits unrestricted non-commercial use, distribution, and reproduction in any medium, provided the original work is properly cited. 


\section{Introduction}

The beach-chair position (BCP), in which the operating table is raised up to $30^{\circ}-90^{\circ}$ from the horizontal plane, is commonly used for shoulder surgery [1]. While the BCP has many advantages, such as an upright and anatomical position, excellent visualization, and simple conversion to an open procedure, if needed, it is also related to high incidences of hypotension and cerebral desaturation events (CDEs), of up to $80 \%$, although serious neurological injuries, such as blindness and brain and spinal cord ischemia, are seen in fewer than $0.1 \%$ of cases [2-4].

Near-infrared spectroscopy (NIRS) has gained popularity as a non-invasive means of estimating cerebral perfusion during surgery through the measurement of cerebral oxygenation. NIRS estimates regional cerebral tissue oxygen saturation $\left(\mathrm{rSO}_{2}\right)$ via transcutaneous measurements with light penetration of the skull, providing real-time estimates of cerebral perfusion. It potentially allows early detection of CDEs and appropriate interventions to increase cerebral perfusion [5].

The sitting position induces a decrease in venous return, resulting in decreases in stroke volume and cardiac output (CO), primarily due to the effect of gravity. This is compensated for by an increase in systemic vascular resistance (SVR) in awake patients, whereas in anesthetized patients, this autonomic response is blocked by vasodilating anesthetics, accompanying the myocardial depressant effect of the anesthetic agents [6]. As a result, the blood pressure remains unchanged or increases slightly in awake patients, but decreases in anesthetized patients in the BCP. Cerebral perfusion pressure (CPP) decreases in the BCP in awake patients by $\sim 15 \%$, and possibly more under general anesthesia for the same reasons as described above.

Previous randomized investigations attempted to reduce the occurrence of hypotension and CDEs via several strategies, such as the use of vasoactive drugs and ventilation methods and devices to increase venous return, although they obtained conflicting results [1,2,7-10]. Compression stockings (CSs), which are widely prescribed for surgical patients to prevent deep vein thrombosis and to improve venous hemodynamics, can be applied readily to patients. Their efficacy has also been studied in patients suffering from spinal cord injury who have sympathetic nerve system dysfunction, such as an inability to induce vasoconstriction below the lesion $[11,12]$. Those studies showed that CSs enhanced sympathetic activity, supported the redistribution of blood, and reduced orthostatic challenge.

To our knowledge, only one study has reported the effects of CSs on CDE in obese patients in the BCP; that study was a prospective case series [2]. We hypothesized that applying thighhigh CSs would attenuate the responses produced by placing patients in the BCP under general anesthesia, such as the decrease in venous return and the lack of an autonomic response. In this randomized prospective study, we sought to determine whether the use of CSs would maintain the value of $\mathrm{rSO}_{2}$ and reduce the incidence of $\mathrm{CDE}$ and hypotension in patients undergoing orthopedic surgery in the BCP.

\section{Materials and Methods}

This prospective and randomized controlled study was conducted between January 2015 and October 2016. After approval of the local ethics committee, patients undergoing elective orthopedic surgery in the BCP under general anesthesia, $\geq 19$ years old, and with American Society of Anesthesiologists physical status I-III were enrolled. Exclusion criteria were patient's refusal, preexisting ischemic cerebrovascular disease, neurological disease, a history of cervical spine surgery, severe lung disease, hypovolemia, uncontrolled hypertension, and any difficulty in wearing CSs on the lower extremities. Written informed consent was obtained from all patients.

Patients were assigned randomly to the control or CS group according to a computer-generated randomization table. On arrival at the operating room, all patients were placed in the supine position. Sensors for NIRS (INVOS ${ }^{\mathrm{TM}} 5100$; Somanetics Corporation, USA) were placed at least $2 \mathrm{~cm}$ above the eyebrow on the right and left sides of the forehead, according to the manufacturer's instructions. Sensors for the bispectral index (BIS Sensor-Aspect Medical Systems, Inc., USA) were applied below them, above the eyebrow. For patients who were scheduled for arthroscopic surgery (all patients) and total shoulder replacement (as requested), interscalene block (ISB) under ultrasound guidance was performed using $12 \mathrm{ml} \mathrm{0.5 \%} \mathrm{ropivacaine}$ before anesthesia induction. After intravenous injection of 0.2 $\mathrm{mg}$ glycopyrrolate and $3 \mathrm{mg}$ midazolam, general anesthesia was induced with propofol (2 mg/kg), fentanyl ( $1 \mu \mathrm{g} / \mathrm{kg})$, and rocuronium $(0.6 \mathrm{mg} / \mathrm{kg})$, followed by tracheal intubation. After anesthesia induction, appropriately sized thigh-high CSs (Venex, micro-light CSs; Heinz Schiebler, Germany) were placed on the patients in the CS group, according to the randomization. Anesthesia was maintained using a 50\% oxygen/air mixture and sevoflurane to maintain the BIS value between 40 and 60 . Ventilation was controlled to maintain end-tidal carbon dioxide $\left(\mathrm{ETCO}_{2}\right)$ between 35 and $40 \mathrm{mmHg}$. The patients were positioned with the back portion of the table angled at $45^{\circ}$, with hip and knee flexed, and remained in that position throughout the operation. The head was secured in a neutral position. Non-invasive blood pressure (NIBP) was obtained from measurements on the non-operative upper arm. Invasive arterial blood pressure was measured through a radial artery cannulation of the non-operative arm, with the pressure transducer zeroed at the external auditory meatus. This estimated arterial blood pressure (eABP) was regarded as representing the arterial pressure at the 
level of the brain. All surgeries were performed by one surgeon.

The $\mathrm{rSO}_{2}$, NIBP, and eABP values were recorded at following times: after preoxygenation before the induction of anesthesia (T0), 5 min after anesthesia was induced and hemodynamics became stable (T1 [baseline]), $3 \mathrm{~min}$ (T2), $5 \mathrm{~min}$ (T3), $1 \mathrm{~h}$ after the BCP (T4), and $5 \mathrm{~min}$ after being placed in a supine position at the end of surgery (T5). When the trend of $\mathrm{rSO}_{2}$ over time was analyzed, the mean values of left and right $\mathrm{rSO}_{2}$ at each time point were used. CDE was defined as a decrease in the $\mathrm{rSO}_{2}$ of $20 \%$ or greater from baseline for $15 \mathrm{~s}$ or more in either hemisphere of the brain. When a CDE developed, we checked head position, blood pressure, $\mathrm{ETCO}_{2}$, depth of anesthesia, fraction of inspired $\mathrm{O}_{2}\left(\mathrm{FIO}_{2}\right)$, and hemoglobin. If the CDE persisted, the anesthesiologist was instructed to treat each CDE with ephedrine in $5 \mathrm{mg}$ increments, a fluid bolus, or both, as required clinically. Hypotension, defined as mean blood pressure $<65 \mathrm{mmHg}$ or a decrease of $20 \%$ or more from baseline, was also treated with $5 \mathrm{mg}$ ephedrine, a fluid bolus, or both, at the discretion of the attending anesthesiologist. For any $\mathrm{CDE}, \mathrm{rSO}_{2}, \mathrm{NIBP}$, eABP, and the intervention administered were recorded at the time of the event.

\section{Statistical analysis}

SPSS software (ver. 18.0; SPSS Inc., USA) was used for statistical analyses. The primary outcome was a difference in the value of $\mathrm{rSO}_{2}$ between groups in the BCP. Secondary outcomes were the incidences of CDE and hypotension, the number of interventions required to treat $\mathrm{CDE}$ or hypotension, and correlations among $\mathrm{rSO}_{2}$, NIBP, and eABP. Based on our previous experience and the report by Lee et al. [13] we expected the value of $\mathrm{rSO}_{2}$ in the BCP to be 67.4 (4.9\%) and a 5\% difference of the absolute value between the two groups was considered clinically significant. A sample size of 17 patients $(\alpha=0.05$, power $=80 \%)$ per group was calculated and allowance for dropouts required 38 patients. Continuous variables were analyzed using the Mann-Whitney $U$ test or Student's $t$ test after an assessment for normality and are presented as mean (SD) or as medians (interquartile range), as appropriate. Discrete variables were analyzed using $\chi^{2}$ or Fisher's exact tests. Changes in $\mathrm{rSO}_{2}$ over time were analyzed using repeated-measures analysis of variance (ANOVA) with the Bonferroni correction. Spearman correlation coefficients were calculated to assess any correlation among $\mathrm{rSO}_{2}$, NIBP, and eABP during a CDE. A P value $<0.05$ was considered to indicate statistical significance.

\section{Results}

In total, 42 patients undergoing orthopedic surgery in the BCP under general anesthesia were assessed for eligibility; of them, 38 were included in the analysis (Fig. 1). There were no differences in demographic data or perioperative clinical details, such as duration of anesthesia or surgery, or type of surgery, between the groups (Table 1).

The repeated-measures ANOVA statistics revealed that the values of $\mathrm{rSO}_{2}, \mathrm{NIBP}$, and eABP did not differ between the groups throughout the surgery and decreased significantly after placing patients in the BCP versus baseline in both groups (Table 2). As shown in Table 3, 36 episodes of CDE occurred in 20 patients. The incidence of CDE was $47.4 \%$ in controls and $57.9 \%$ in the CS group, with no significant difference between them. The total number of episodes of CDE in each group was also similar.

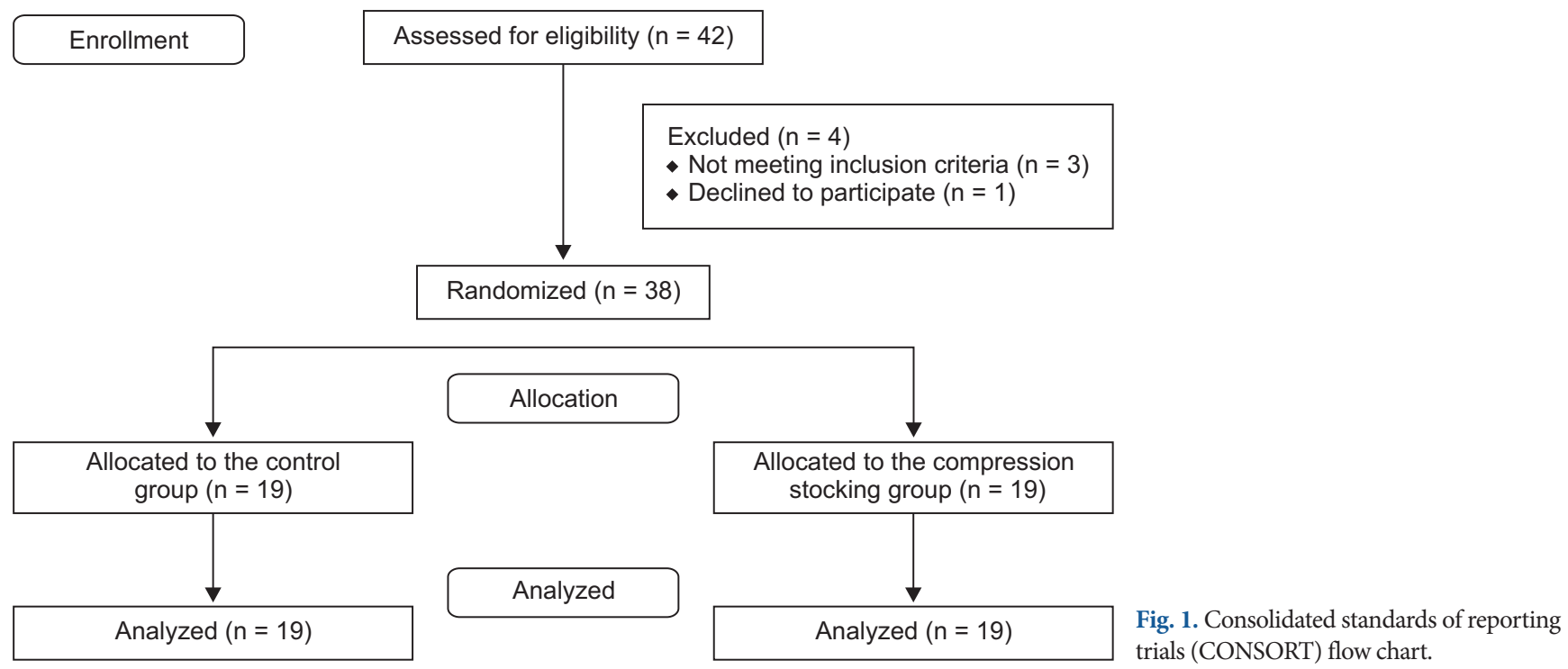

Online access in http://ekja.org 
The incidence of hypotension, measured by eABP, was significantly lower in patients wearing CSs versus controls $(\mathrm{P}=0.020)$, whereas this difference was not statistically significant when measured by NIBP. The numbers of hypotension events detect-

Table 1. Demographic and Intraoperative Variables

\begin{tabular}{lcc}
\hline & Control $(\mathrm{n}=19)$ & Stocking $(\mathrm{n}=19)$ \\
\hline Gender $(\mathrm{M} / \mathrm{F})$ & $14 / 5$ & $14 / 5$ \\
Age $(\mathrm{yr})$ & $47(20)$ & $48(15)$ \\
Height $(\mathrm{cm})$ & $170.4(9.7)$ & $167.2(9.0)$ \\
Weight $(\mathrm{kg})$ & $71.3(11.8)$ & $70.1(10.2)$ \\
ASA (I/II) & $7 / 12$ & $9 / 10$ \\
Surgery & & \\
Total shoulder replacement & $4(21.1 \%)$ & $2(10.5 \%)$ \\
Arthroscopic shoulder surgery & $5(26.3 \%)$ & $5(26.3 \%)$ \\
Clavicle fracture ORIF & $6(31.6 \%)$ & $11(57.9 \%)$ \\
$\quad$ Humerus fracture ORIF & $4(21.1 \%)$ & $1(5.3 \%)$ \\
Interscalene block & $7(36.8 \%)$ & $6(31.6 \%)$ \\
Diabetes & $2(10.5 \%)$ & $2(10.5 \%)$ \\
Hypertension & $3(15.8 \%)$ & $5(26.3 \%)$ \\
Mean NIBP at T0 (mmHg) & $96(89,102)$ & $100(91,105)$ \\
Mean rSO ${ }_{2}$ at T0 (\%) & $78(72.5,83)$ & $75(69.5,78.5)$ \\
Duration of anesthesia (min) & $164.4(38.5)$ & $167.1(29.5)$ \\
Duration of surgery (min) & $109.4(33.2)$ & $106.8(27.3)$ \\
Duration of beach chair & $123.0(38.8)$ & $124.3(27.0)$ \\
position (min) & & \\
Fluid administration (ml) & $1100(800,1400)$ & $900(850,1030)$ \\
Blood loss (ml) & $100(10,300)$ & $80(10,250)$ \\
\hline
\end{tabular}

Data are presented as number (\%), mean (SD) or median (interquartile range). ASA: American Society of Anesthesiologists, ORIF: open reduction and internal fixation, NIBP: non-invasive blood pressure, T0: after preoxygenation before the induction of anesthesia, $\mathrm{rSO}_{2}$ : regional cerebral tissue oxygen saturation. ed by NIBP or eABP in each patient also differed significantly between groups $(\mathrm{P}=0.018)$. More patients in the control group experienced hypotension more than once. The numbers of patients receiving interventions to treat hypotension or CDE were similar between the groups: 14 (73.7\%) in the controls and 12 (63.2\%) in the CS group. Of 14 patients receiving interventions in the control group, 13 (92.9\%) had hypotension, whereas 8 of $12(66.7 \%)$ patients in the CS group showed CDE without hypotension. The ephedrine dose used during surgery did not differ between the two groups.

The $\mathrm{rSO}_{2}$, NIBP, and eABP values in $36 \mathrm{CDE}$ episodes were analyzed (Table 4). CDEs developed only during the BCP. Compared with baseline, the values of $\mathrm{rSO}_{2}, \mathrm{NIBP}$, and eABP decreased significantly when CDE occurred in both groups. The value of NIBP at the time of CDE was significantly higher in patients wearing CSs $(\mathrm{P}=0.019)$ than in the control group. No significant correlation was found between NIBP and $\mathrm{rSO}_{2}\left(\mathrm{r}_{\mathrm{s}}=\right.$ -0.208; $\mathrm{P}=0.224)$ or between eABP and $\mathrm{rSO}_{2}\left(\mathrm{r}_{\mathrm{s}}=-0.251 ; \mathrm{P}=\right.$ 0.139) during CDEs (Fig. 2). Regarding NIBP and eABP, there was a significant correlation $\left(r_{s}=0.757 ; \mathrm{P}<0.001\right)$. No adverse events, including neurological sequelae, were reported postoperatively.

\section{Discussion}

Our results show that use of thigh-high CSs reduced the incidence of hypotension significantly, but this did not contribute to a reduction in CDE occurrence in patients undergoing orthopedic surgery in the BCP. CDE did not always arise from hypotension. Changes in NIBP and eABP did not correlate with changes in $\mathrm{rSO}_{2}$. Whereas the interventions in the control group were

Table 2. Values of $\mathrm{rSO}_{2}$, NIBP, and eABP during Surgery in All Patients

\begin{tabular}{lccccc}
\hline & $\mathrm{T} 1$ & $\mathrm{~T} 2$ & $\mathrm{~T} 3$ & $\mathrm{~T} 4$ & $\mathrm{~T}$ \\
\hline $\mathrm{rSO}_{2}(\%)$ & & & & \\
Overall & & & & \\
Control & $77.5(71.9,84.6)$ & $67(61.13,74.1)^{*}$ & $66.5(60.9,74.5)^{*}$ & $67.5(59.9,73.8)^{*}$ & $71(62.9,80.3)^{*}$ \\
$\quad$ Stocking & $79(72,87.5)$ & $67(62.5,78.5)^{*}$ & $68(62.5,76)^{*}$ & $69(59.5,75)^{*}$ & $74.5(68,87)$ \\
Mean NIBP (mmHg) & $77(69,83.5)$ & $67(60,73)^{*}$ & $66(60.5,72.5)^{*}$ & $66(60,71.5)^{*}$ & $67(61.5,76)$ \\
Overall & & & & \\
Control & $101(92.5,110)$ & $86.5(75,98.3)^{*}$ & $81.5(68.8,93.5)^{*}$ & $87(75.25,100.3)^{*}$ & $96(85,107.3)$ \\
Stocking & $100(86,110)$ & $81(65,99)^{*}$ & $80(65,93)^{*}$ & $82(69,94)^{*}$ & $95(85,104)$ \\
Mean eABP (mmHg) & $103(94,108)$ & $89(80,98)^{*}$ & $85(75,95)^{*}$ & $89(78,105)$ & $100(85,108)$ \\
Overall & $100(90.8,105.3)$ & $76.5(64.5,84.3)^{*}$ & $72.5(65.8,80.5)^{*}$ & $80(70,91.25)^{*}$ & $100(93,104.3)$ \\
Control & $99(88,105)$ & $76(63,82)^{*}$ & $75(67,79)^{*}$ & $78(70,90)^{*}$ & $100(95,112)$ \\
Stocking & $100(95,106)$ & $78(65,87)^{*}$ & $70(65,84)^{*}$ & $84(70,93)^{*}$ & $96(85,101)$ \\
\hline
\end{tabular}

Data are presented as median (interquartile range). The values of $\mathrm{rSO}_{2}$, NIBP, and eABP decreased significantly after placing patients in the beachchair position versus baseline in both groups, while those values did not differ between the groups throughout the surgery. $\mathrm{rSO}_{2}$ : regional cerebral tissue oxygen saturation, NIBP: non-invasive blood pressure, eABP: invasive arterial blood pressure zeroed at the external auditory meatus, T1: 5 min after the anesthesia induction and hemodynamics became stable, T2: $3 \mathrm{~min}, \mathrm{~T} 3: 5 \mathrm{~min}, \mathrm{~T} 4: 1 \mathrm{~h}$ after the beach-chair position, T5: $5 \mathrm{~min}$ after a supine position at the end of surgery. ${ }^{*} \mathrm{P}<0.05$ versus baseline. 
Table 3. Incidence of Cerebral Desaturation Events and Hypotension

\begin{tabular}{|c|c|c|c|}
\hline & Control $(\mathrm{n}=19)$ & Stocking $(\mathrm{n}=19)$ & $P$ value \\
\hline $\mathrm{CDE}$ & $9(47.4 \%)$ & $12(63.2 \%)$ & 0.257 \\
\hline The number of experiencing CDE in each patient & & & 0.414 \\
\hline 1 & $7(36.8 \%)$ & $6(31.6 \%)$ & \\
\hline 2 & $0(0 \%)$ & $3(15.8 \%)$ & \\
\hline 3 & $1(5.3 \%)$ & $2(10.5 \%)$ & \\
\hline 4 & $1(5.3 \%)$ & $1(5.3 \%)$ & \\
\hline Total & 14 & 22 & \\
\hline Hypotension by NIBP & $9(47.4 \%)$ & $3(15.8 \%)$ & 0.079 \\
\hline Hypotension by eABP & $12(63.2 \%)$ & $4(21.1 \%)$ & 0.020 \\
\hline The number of experiencing hypotension in each patient* & & & 0.018 \\
\hline 1 & $5(26.3 \%)$ & $3(15.8 \%)$ & \\
\hline 2 & $5(26.3 \%)$ & $1(5.3 \%)$ & \\
\hline 3 & $3(15.8 \%)$ & $0(0 \%)$ & \\
\hline The number of patients receiving intervention due to hypotension or CDE & $14(73.7 \%)$ & $12(63.2 \%)$ & 0.728 \\
\hline Intervention to treat hypotension \& $\mathrm{CDE}$ simultaneously & $8(42.1 \%)$ & $4(21.1 \%)$ & 0.295 \\
\hline Intervention to treat $\mathrm{CDE}$ without hypotension & $1(10.5 \%)$ & $8(42.1 \%)$ & 0.019 \\
\hline Intervention to treat hypotension without $\mathrm{CDE}$ & $5(26.3 \%)$ & $0(0 \%)$ & 0.046 \\
\hline Ephedrine (mg) & $5(0,10)$ & $0(0,5)$ & 0.311 \\
\hline
\end{tabular}

Data are presented as number (\%) or median (interquartile range). CDE: cerebral desaturation event, NIBP: non-invasive blood pressure, eABP: invasive arterial blood pressure zeroed at the external auditory meatus. ${ }^{*}$ Hypotension detected by NIBP or eABP.

Table 4. $\mathrm{rSO}_{2}$, NIBP, and eABP Values at the Time of CDE

\begin{tabular}{|c|c|c|c|c|c|c|c|c|}
\hline & \multicolumn{4}{|c|}{ Control $(\mathrm{n}=9)$} & \multicolumn{4}{|c|}{ Stocking $(\mathrm{n}=12)$} \\
\hline & Baseline & $\mathrm{CDE}$ & $\%$ change & $P$ value & Baseline & $\mathrm{CDE}$ & $\%$ change & $P$ value \\
\hline $\begin{array}{r}\mathrm{rSO}_{2} \\
(\%)\end{array}$ & $\begin{array}{c}73 \\
(59,76.5)\end{array}$ & $\begin{array}{c}52 \\
(43.8,58.5)\end{array}$ & $\begin{array}{c}-26.4 \\
(-29.2,-22.9)\end{array}$ & $<0.001$ & $\begin{array}{c}77 \\
(70,80.8)\end{array}$ & $\begin{array}{c}54 \\
(50.8,57.3)\end{array}$ & $\begin{array}{c}-28.8 \\
(-33.1,-22.5)\end{array}$ & $<0.001$ \\
\hline $\begin{array}{c}\text { Mean NIBP } \\
(\mathrm{mmHg})\end{array}$ & $\begin{array}{c}95.5 \\
(90,101)\end{array}$ & $\begin{array}{c}71.5 \\
(62.8,83)\end{array}$ & $\begin{array}{c}-23.3 \\
(-31.6,-17.8)\end{array}$ & $<0.001$ & $\begin{array}{c}95 \\
(90.5,99.5)\end{array}$ & $\begin{array}{c}85^{*} \\
(65,100)\end{array}$ & $\begin{array}{c}-14.8 \\
(-24.6,-4.2)\end{array}$ & 0.035 \\
\hline $\begin{array}{l}\text { Mean eABP } \\
(\mathrm{mmHg})\end{array}$ & $\begin{array}{c}95 \\
(88,109)\end{array}$ & $\begin{array}{c}66.5 \\
(59.8,78)\end{array}$ & $\begin{array}{c}-28.4 \\
(-37.1,-27.9)\end{array}$ & $<0.001$ & $\begin{array}{c}100 \\
(86.5,100)\end{array}$ & $\begin{array}{c}72 \\
(65,92)\end{array}$ & $\begin{array}{c}-11.8 \\
(-33.9,-6.5)\end{array}$ & 0.003 \\
\hline
\end{tabular}

Data are presented as median (interquartile range). $\mathrm{rSO}_{2}$ : regional cerebral tissue oxygen saturation, NIBP: non-invasive blood pressure, eABP: invasive arterial blood pressure zeroed at the external auditory meatus, $\mathrm{CDE}$ : cerebral desaturation event. ${ }^{*} \mathrm{P}=0.019$ versus control group.

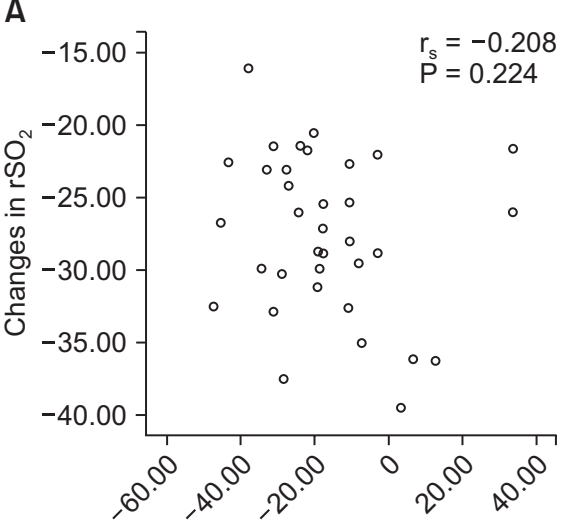

Changes in NIBP
B

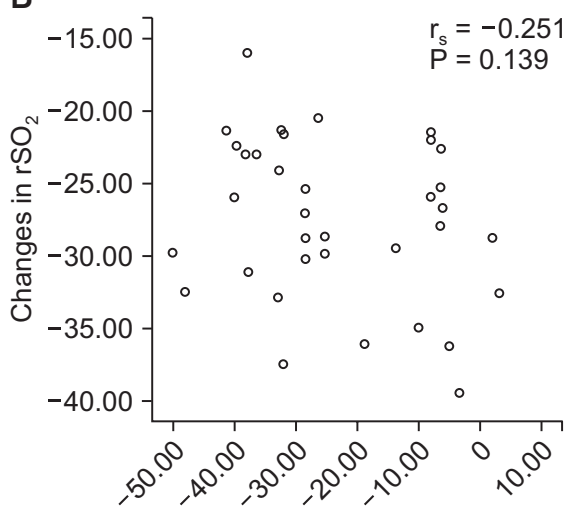

Changes in eABP

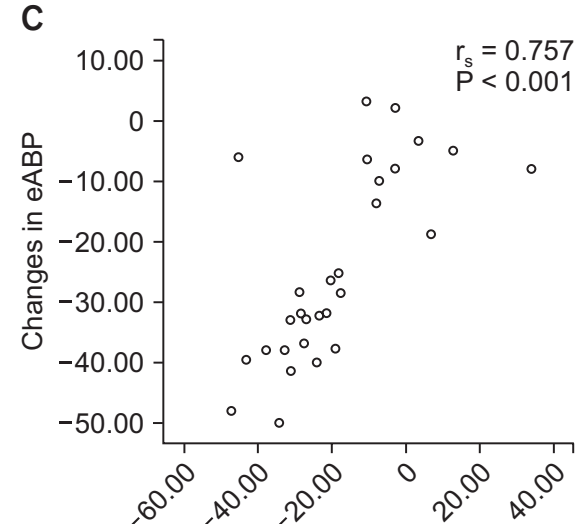

Changes in NIBP

Fig. 2. Correlations between (A) NIBP and $\mathrm{rSO}_{2}$, (B) eABP and $\mathrm{rSO}_{2}$, and (C) NIBP and eABP. During 36 cerebral desaturation event episodes, no significant correlation was found between NIBP and $\mathrm{rSO}_{2}(\mathrm{P}=0.224)$ or between eABP and $\mathrm{rSO}_{2}(\mathrm{P}=0.139)$, while there was a significant correlation between NIBP and eABP $(\mathrm{P}<0.001)$. $\mathrm{rSO}_{2}$ : regional cerebral tissue oxygen saturation, NIBP: non-invasive blood pressure, eABP: invasive arterial blood pressure zeroed at the external auditory meatus. 
mostly related to hypotension, $67 \%$ of patients in the CS group showed CDEs even though blood pressure was maintained within the predefined range.

Cerebrovascular risk factors, such as advanced age, male gender, hypertension, diabetes, coronary artery disease, carotid artery disease, and atrial fibrillation, have been described in the general population. However, major brain and spinal cord injuries during surgery in the upright position have been reported even in healthy patients seemingly at extremely low risk for cerebrovascular events $[14,15]$. The etiology of CDE is not fully understood. It has been suggested that central blood volume may be more vulnerable to loss of peripheral resistance and CO decreases when in the sitting position $[16,17]$. Several factors behind these events, such as anatomical variables like external compression of carotid and vertebral vessels by inappropriate head positioning and cervical osteophytes, anomalies in vessel configuration, and intraluminal atherosclerosis, have also been proposed [14,17]. However, considering that the incidence of $\mathrm{CDE}$ under regional anesthesia was significantly lower than that under general anesthesia in a previous study [18], CDE would seem to be attributable primarily to the effects of general anesthetics rather than these anatomical variables. $\mathrm{CDE}$ in the BCP under regional anesthesia was observed in only $0.77 \%$ of patients, despite hypotension being common (as high as 76\%) [18].

CSs are a readily applicable medical product, requiring no special device. In this study, we assumed that the use of thighhigh CSs would help to reduce venous pooling in the lower extremities and maintain venous tone, in turn preventing the occurrence of hypotension and CDEs. Our results show that CSs reduced the incidence of hypotension, but not that of CDEs. In contrast to our results, in a previous prospective case series with historical control of obese patients (mean body mass index of $35.39 \mathrm{~kg} / \mathrm{m}^{2}$ ), the use of thigh-high CSs was shown to decrease the incidence of CDEs ( $4 \%$ in the CS group versus $18 \%$ in controls) in the BCP [2]. However, that study had some limitations, such as the low level of evidence of the study design, uneven distribution of characteristics between the groups, and no available intraoperative hemodynamic data. They also applied additional bilateral sequential compression devices to all patients, which could be a confounding factor in determining the value of CSs.

In this study, we standardized the anesthesia protocol, using the same anesthetic regimen and ventilation strategies, such as $\mathrm{FIO}_{2}$ and $\mathrm{ETCO}_{2}$ control, which can all influence $\mathrm{rSO}_{2}$ values. Only in 13 patients undergoing arthroscopic shoulder surgery and total shoulder replacement, we performed ISB using $0.5 \%$ ropivacaine before general anesthesia induction for postoperative analgesia, as per the surgeon's request. ISB can induce a block of the cervical sympathetic chain, which can affect cerebral blood flow. In a study comparing $\mathrm{rSO}_{2}$ of unblocked and blocked sides using $25-30 \mathrm{ml} 0.75 \%$ ropivacaine, $\mathrm{rSO}_{2}$ on the blocked side did not differ from that on the other side, despite the presence of Horner's syndrome [19]. The volume used for ISB $(12 \mathrm{ml})$ in our institution is much lower than that used in their study. We consider it unlikely that the use of this block made a difference in the incidence of hypotension or CDE. The number of patients receiving ISB was evenly distributed between the two groups.

The lower limit of cerebral autoregulation is known to be a mean arterial pressure of $50 \mathrm{mmHg}$ to as high as $90 \mathrm{mmHg}$ in awake, normotensive subjects. According to the waterfall theory, a higher NIBP threshold at the level of the arm is required to maintain CPP. For estimating CPP in the BCP, invasive arterial monitoring, with the transducer zeroed at the external auditory meatus, has been used commonly. Triplet et al. [20] described that the $\mathrm{ABP}$ to NIBP ratio, measured at the level of the upper arm, declined as the tilting angle increased and concluded that eABP could be helpful as a surrogate for CPP. In this study, we defined hypotension as mean blood pressure $<65 \mathrm{mmHg}$ or $20 \%$ or more higher than baseline and the measurement of eABP, but not NIBP, detected a significantly different incidence of hypotension between the groups. Although eABP can help to prevent the overestimation of CPP from measuring NIBP, it is unlikely that CDEs are always caused by low eABP or NIBP. Our results show that CDEs persisted when blood pressure was within the normal range, or even above in some patients. One of the likely causes of this is that the cerebral cortex is located above the position of the external auditory meatus, so an even higher arterial pressure may be required to maintain the CPP [21]. Lesions in cases reporting catastrophic neurological events varied from the cerebral cortex, the cortical thalamic region, and midbrain, to the upper spinal cord.

It has recently been suggested that to estimate appropriate cerebral perfusion blood pressure per se is less important than CO. Previous studies also observed an inconsistency between hypotension and CDEs occurrence [4,20]. Triplet et al. [20] demonstrated that neither NIBP nor mean arterial pressure measured at the level of the temporal bone correlated with decreases in $\mathrm{rSO}_{2}$ in the BCP. In another study comparing the BCP and a lateral position, there was no difference in mean arterial pressure despite a much higher incidence of CDEs [4]. It has also been shown that vasopressin and phenylephrine increase blood pressure but reduce $\mathrm{CO}$ and $\mathrm{rSO}_{2}$ under general anesthesia, while ephedrine increases $\mathrm{CO}$ and maintains cerebral oxygenation $[7,22]$. This indicates that blood pressure measurements alone might not be useful in predicting the occurrence of CDEs and that other factors beyond blood pressure contribute to CDEs in the BCP. These might include $\mathrm{CO}, \mathrm{ETCO}_{2}$, cerebral metabolism, intravascular volume status, intraluminal atherosclerosis, and anatomical variables, as mentioned above [17]. Among these, some variables can be adjusted to improve patient outcomes. 
Schramm et al. [23] described that $\mathrm{rSO}_{2}$ even increased slightly in the sitting position for neurosurgical surgery, which contrasts with our and previous studies regarding the BCP in orthopedic surgery. Schramm et al. [23] focused on the maintenance of CO with transesophageal echocardiographic monitoring and gave a fluid bolus of $500 \mathrm{ml}$ of colloid before changing to a sitting position, differing from the present study design. They concluded that $\mathrm{rSO}_{2}$ correlated with $\mathrm{CO}$ rather than blood pressure, which is consistent with our findings, and adequate fluid administration was necessary to compensate for venous pooling in the lower extremities and to optimize CO. In the present study, we cannot say how the use of CSs affected CO and SVR because we did not measure these latter two variables, which would have required advanced cardiovascular monitoring, such as transesophageal echocardiography, thermodilution, or invasive arterial waveform shape analysis, and additional central venous pressure monitoring. We think it possible that intravascular volume might not be sufficient to optimize CO and SVR as a result of the benefit of CSs after changing to the BCP, which could underestimate the effect of CSs. We did not administer as much fluid as in neurosurgical patients because the orthopedic surgeries in this study commonly require less time and have lower estimated blood loss. Sufficient fluid administration with the use of CSs may lead to different results. Further study with advanced hemodynamic monitoring is required to define the etiology of CDE with efforts to reduce hypotension and CDE in the BCP.

Our study has several limitations. First, our sample size was small and we did not use advanced hemodynamic monitoring. Thus, as noted above, we cannot reach a conclusion regarding the etiology, as to why CDEs developed despite the low incidence of hypotension in the CS group. Second, our study focused on CDEs, which develop quite often in the BCP, not on 'real' cerebrovascular events. It is unclear whether $\mathrm{rSO}_{2}$ values are directly connected with cerebrovascular events. In numerous studies investigating CDEs in the BCP, no patient developed a cerebrovascular event despite a high incidence of CDEs. Interventions to try to increase oxygen delivery to tissues, such as adjusting $\mathrm{FIO}_{2}$ and $\mathrm{ETCO}_{2}$ control, have actually been shown to increase $\mathrm{rSO}_{2}$. In contrast, we could not determine from the literature exactly how much of a decrease from the baseline $\mathrm{rSO}_{2}$ results in catastrophic cerebrovascular events, such as infarction. Nevertheless, as many studies in cardiac and major abdominal surgery have demonstrated that detection of CDEs results in early treatment and better clinical outcomes, it is generally accepted that an effort to monitor cerebral oxygenation to reduce CDEs is required in susceptible patients [24-26].

In conclusion, the use of thigh-high CSs reduced the incidence of hypotension, but this did not contribute to a reduction in the occurrence of CDEs in patients undergoing orthopedic surgery in the BCP. Our results indicate that there are other factors, beyond hypotension, that contribute to CDE in the BCP under general anesthesia. Further study using advanced hemodynamic monitoring is warranted to clarify the mechanism of CDEs in the BCP.

\section{ORCID}

Jae Hee Woo, https://orcid.org/0000-0002-1993-1687

Youn Jin Kim, https://orcid.org/0000-0001-9189-5839

\section{References}

1. Picton P, Dering A, Alexander A, Neff M, Miller BS, Shanks A, et al. Influence of ventilation strategies and anesthetic techniques on regional cerebral oximetry in the beach chair position: a prospective interventional study with a randomized comparison of two anesthetics. Anesthesiology 2015; 123: 765-74.

2. Tauchen AJ, Salazar D, Barton GJ, Francois A, Tonino P, Garbis NG, et al. The effect of compression stockings on cerebral desaturation events in obese patients undergoing shoulder arthroscopy in the beach-chair position. Arthroscopy 2015; 31: 2352-64.

3. Friedman DJ, Parnes NZ, Zimmer Z, Higgins LD, Warner JJ. Prevalence of cerebrovascular events during shoulder surgery and association with patient position. Orthopedics 2009; 32: 256.

4. Murphy GS, Szokol JW, Marymont JH, Greenberg SB, Avram MJ, Vender JS, et al. Cerebral oxygen desaturation events assessed by nearinfrared spectroscopy during shoulder arthroscopy in the beach chair and lateral decubitus positions. Anesth Analg 2010; 111: 496-505.

5. Murkin JM, Arango M. Near-infrared spectroscopy as an index of brain and tissue oxygenation. Br J Anaesth 2009; 103 Suppl 1: i3-13.

6. Porter JM, Pidgeon C, Cunningham AJ. The sitting position in neurosurgery: a critical appraisal. Br J Anaesth 1999; 82: 117-28.

7. Cho SY, Kim SJ, Jeong CW, Jeong CY, Chung SS, Lee J, et al. Under general anesthesia arginine vasopressin prevents hypotension but impairs cerebral oxygenation during arthroscopic shoulder surgery in the beach chair position. Anesth Analg 2013; 117: 1436-43.

8. Kwak HJ, Lee JS, Lee DC, Kim HS, Kim JY. The effect of a sequential compression device on hemodynamics in arthroscopic shoulder surgery using beach-chair position. Arthroscopy 2010; 26: 729-33.

9. Kim JY, Lee JS, Lee KC, Kim HS, Park CH, Kwak HJ. The effect of sequential compression device on hypotension in the sitting position during shoulder arthroscopy; a comparison with elastic stocking. Korean J Anesthesiol 2009; 57: 417-21. 
10. Soeding PF, Hoy S, Hoy G, Evans M, Royse CF. Effect of phenylephrine on the haemodynamic state and cerebral oxygen saturation during anaesthesia in the upright position. Br J Anaesth 2013; 111: 229-34.

11. Rimaud D, Calmels P, Pichot V, Bethoux F, Roche F. Effects of compression stockings on sympathetic activity and heart rate variability in individuals with spinal cord injury. J Spinal Cord Med 2012; 35: 81-8.

12. Rimaud D, Calmels P, Roche F, Mongold JJ, Trudeau F, Devillard X. Effects of graduated compression stockings on cardiovascular and metabolic responses to exercise and exercise recovery in persons with spinal cord injury. Arch Phys Med Rehabil 2007; 88: 703-9.

13. Lee JH, Min KT, Chun YM, Kim EJ, Choi SH. Effects of beach-chair position and induced hypotension on cerebral oxygen saturation in patients undergoing arthroscopic shoulder surgery. Arthroscopy 2011; 27: 889-94.

14. Pohl A, Cullen DJ. Cerebral ischemia during shoulder surgery in the upright position: a case series. J Clin Anesth 2005; 17: 463-9.

15. Bhatti MT, Enneking FK. Visual loss and ophthalmoplegia after shoulder surgery. Anesth Analg 2003; 96: 899-902.

16. So J, Shin WJ, Shim JH. A cardiovascular collapse occurred in the beach chair position for shoulder arthroscopy under general anesthesia -a case report-. Korean J Anesthesiol 2013; 64: 265-7.

17. Dippmann C, Winge S, Nielsen HB. Severe cerebral desaturation during shoulder arthroscopy in the beach-chair position. Arthroscopy 2010; 26(9 Suppl): S148-50.

18. Yadeau JT, Liu SS, Bang H, Shaw PM, Wilfred SE, Shetty T, et al. Cerebral oximetry desaturation during shoulder surgery performed in a sitting position under regional anesthesia. Can J Anaesth 2011; 58: 986-92.

19. Soeding PF, Currigan DA, Mamo Y, Hoy G. Effect of interscalene anaesthesia on cerebral oxygen saturation. Anaesth Intensive Care 2016; 44: 359-63.

20. Triplet JJ, Lonetta CM, Levy JC, Everding NG, Moor MA. Cerebral desaturation events in the beach chair position: correlation of noninvasive blood pressure and estimated temporal mean arterial pressure. J Shoulder Elbow Surg 2015; 24: 133-7.

21. Nociti JR. Positioning of the anesthetized patient and cerebral perfusion: foreseeable catastrophes. Rev Bras Anestesiol 2008; 58: 1-4.

22. Meng L, Cannesson M, Alexander BS, Yu Z, Kain ZN, Cerussi AE, et al. Effect of phenylephrine and ephedrine bolus treatment on cerebral oxygenation in anaesthetized patients. Br J Anaesth 2011; 107: 209-17.

23. Schramm P, Tzanova I, Hagen F, Berres M, Closhen D, Pestel G, et al. Cerebral oxygen saturation and cardiac output during anaesthesia in sitting position for neurosurgical procedures: a prospective observational study. Br J Anaesth 2016; 117: 482-8.

24. Casati A, Fanelli G, Pietropaoli P, Proietti R, Tufano R, Danelli G, et al. Continuous monitoring of cerebral oxygen saturation in elderly patients undergoing major abdominal surgery minimizes brain exposure to potential hypoxia. Anesth Analg 2005; 101: 740-7.

25. Slater JP, Guarino T, Stack J, Vinod K, Bustami RT, Brown JM 3rd, et al. Cerebral oxygen desaturation predicts cognitive decline and longer hospital stay after cardiac surgery. Ann Thorac Surg 2009; 87: 36-44.

26. Suehiro K, Okutai R. Duration of cerebral desaturation time during single-lung ventilation correlates with mini mental state examination score. J Anesth 2011; 25: 345-9. 